\title{
Ophiostomatales (Ascomycètes) associées à Ips acuminatus Gyll (Coleoptera : Scolytidae) sur le pin sylvestre (Pinus sylvestris $L$ ) dans le Sud-Est de la France et comparaison avec Ips sexdentatus Boern
}

\author{
F Lieutier 1, J Garcia 1, A Yart 1, G Vouland 2, M Pettinetti 2, M Morelet 3 \\ 1 INRA, station de zoologie forestière, Ardon 45160 Olivet; \\ 2 INRA, station de zoologie forestière, avenue A Vivaldi, 84000 Avignon; \\ 3 INRA, station de pathologie forestière, Champenoux, 54280 Seichamps, France
}

(Reçu le 24 avril 1991; accepté le 12 août 1991)

\begin{abstract}
Résumé - Les Ophiostomatales associées à lps acuminatus ont été isolées des insectes, de leurs galeries et de l'aubier des pins sylvestres attaqués, dans le Sud-Est de la France (département du Var), pendant l'épidémie de 1987 et 1988. Des isolements ont aussi été réalisés dans la même région en 1987 sur des populations endémiques d'/ps sexdentatus, et dans 2 autres régions (''Orléanais (1988) et le Mont Ventoux (1989)) sur des populations endémiques d'/ acuminatus. Les vitesses de croissance des champignons varois ont été comparées au laboratoire sur malt-agar. Leur agressivité a été appréciée par inoculations artificielles à des pins sylvestres, et mesurée par l'importance du développement de la réaction résinique induite et par l'importance de l'extension des champignons dans cette réaction. Le cortège fongique associé à l acuminatus dans le Sud-Est de la France comprend 4 espèces d'Ophiostomatales et diffère profondément de celui associé aux populations scandinaves et allemandes du même insecte. II diffère également de celui associé à I sexdentatus, même quand les 2 scolytides évoluent sur les mêmes arbres, bien que 2 espèces de champignons (Ophiostoma brunneo-ciliatum et Ophiostoma ips) soient communes aux 2 insectes. Dans le Var, la fréquence d'association avec $l$ acuminatus et le taux de fructification des champignons dans les galeries sont nettement supérieurs en 1987 à ceux de 1988. Cette dernière année, aucune différence n'est observée entre le Var et les régions où les populations sont endémiques. La fréquence d'association est aussi significativement plus faible chez les insectes essaimants que chez ceux dont linstallation a réussi. Des 4 espèces associées à $l$ acuminatus, $O$ ips et $O$ brunneo-ciliatum se montrent les plus agressives après inoculation des arbres. Après l'attaque d'l acuminatus dans la moitié supérieure de l'arbre, le bleuissement de l'aubier semble s'étendre vers la partie inférieure en envahissant d'abord les zones les plus profondes de ce tissu.
\end{abstract}

Ips acuminatus / Ips sexdentatus / Pinus sy/vestris = pin sylvestre / Ophiostoma / Ceratocystiopsis / association scolytide-champignon / relation conifère-scolytide / réaction de défense de l'arbre / agressivité / bleuissement de l'aubier

Summary - Ophiostomatales (Ascomycetes) associated with Ips acuminatus Gyll (Coleoptera: Scolytidae) in Scots pine (Pinus sylvestris L) in south-eastern France, and comparison with Ips sexdentatus Boern. Ophiostomatales associated with Ips acuminatus were isolated from beetles, their galleries and the sapwood of the attacked Scots pines, in south-eastern France (department of the Var), during outbreaks in 1987 and 1988. In 2 other regions, (Orléanais and Mont Ventoux), fungi were isolated from endemic populations of the same beetles in 1988 and 1989. Fungi were also isolated from endemic populations of Ips sexdentatus in the department of the Var in 1987. The growth of the fungi isolated from the Var samples was analysed on malt-agar medium. Their agressiveness was determined after artificial inoculation of Scots pines, by measuring the development of the induced defense reaction (length and resin quantity) and the extension of the fungi within this reaction.

The Ophiostomatales associated with I acuminatus in south-eastern France included 4 species that differed greatly from the Scandinavian and German flora associated with the same insect. They also differed from the flora associated with I sexdentatus, even when the 2 beetles occurred in the same trees, and although the 2 fungi (Ophiostoma Ips and Ophiostoma brunneo-ciliatum) were both associated with the 2 insect species (table I). In the Var region, the fre-quency of the association with I acuminatus, and the fructification rate of the fungi in the galleries were higher in 1987 than in 1988. In 1988, there were no differences between the Var and regions where the beetle populations were en- 
demic. The frequency of association was also significantly lower in the swarming beetles than in the successful attacks (table I). Among the 4 fungus species associated with I acuminatus, $\mathrm{O}$ ips and $\mathrm{O}$ brunneo-ciliatum were the most aggressive after inoculation of trees (fig 2). After the successful attacks of I acuminatus in the upper half of the trees, the blue-stain invaded the lower half, beginning with the innermost zones of the sapwood.

Ips acuminatus / Ips sexdentatus / Pinus sylvestris = Scots pine / Ophiostoma / Ceratocystiopsis / fungusbark beetle association / conifer-bark beetle relationship / tree defense reaction / aggressiveness / sapwood blue-stain

\section{INTRODUCTION}

La plupart des Scolytides des Conifères sont associés à des champignons phytopathogènes, principalement du genre Ophiostoma (FranckeGrosmann, 1963a, 1967; Graham, 1967; Withney, 1982; Solheim, 1986). En Europe, les travaux sur les relations Conifères-Scolytides et le rôle des champignons phytopathogènes dans ces interactions ont porté jusqu'à présent sur Ips typographus L et l'épicéa (Christiansen et Horntvedt, 1983; Horntvedt et al, 1983; Christiansen, 1985), ainsi que sur Tomicus piniperda $L$ et Ips sexdentatus Boern sur le pin sylvestre (Lieutier et al, 1988b, 1989a, b; Langström et al, 1990; Solheim et Langström, 1991). Le plus souvent, on a attribué aux champignons Ophiostoma un rôle important dans les mécanismes d'installation des populations de Scolytides et, dans certains cas, ils ont été rendus responsables de la mort des arbres attaqués. Ips acuminatus Gyll, autre espèce d'intérêt économique, n'a pas jusqu'à présent donné lieu à une telle approche. On ne possède actuellement qu'un inventaire des pathogènes isolés des galeries de ce Scolytide dans différentes conditions, ainsi que des indications sur le mode de transport de certains d'entre eux (Rennerfelt, 1950; FranckeGrosmann, 1952, 1963b, 1967; MathiesenKäärik, 1953; Henningsson et Lundström, 1974).

Les dégâts occasionnés depuis 15 ans par I acuminatus dans les forêts de pins sylvestres françaises, dans la région Centre de 1974 à 1977, puis plus récemment dans le Sud-Est de 1986 à 1988, ont néanmoins suscité un nouvel intérêt pour ce ravageur. Des recherches concernant les interrelations entre $I$ acuminatus, le pin sylvestre et les champignons du groupe des Ophiostomatales ont été développées à partir de 1987.

Nous avons précédemment exposé les problèmes posés par ce Scolytide dans le Sud-Est de la France (biologie et dégâts) et décrit les réactions de défense des arbres attaqués. Nous avons présenté en même temps les méca- nismes susceptibles d'être intervenus dans le déclenchement de la pullulation en 1986 , ou pouvant être responsables de la mort des pins, en nous appuyant sur les résultats obtenus chez les autres espèces de Scolytides du pin sylvestre (Lieutier et al, 1988a). Nous nous proposons ici de présenter un inventaire et quelques caractéristiques biologiques des champignons Ophiostomatales associés à $/$ acuminatus dans cette région, ainsi que les fréquences d'association entre ces microorganismes et leur insecte hôte. En plus des informations que cette étude pouvait apporter pour la compréhension du dépérissement du pin sylvestre dans le Sud-Est de la France, il était intéressant de comparer l'association I acuminatus - Ophiostoma avec l'association I sexdentatus - Ophiostoma étudiée précédemment Lieutier et al, 1988b, 1989a, b). Les 2 espèces de Scolytides ont en effet des caractéristiques biologiques extrêmement voisines (cycle biologique, optimum thermique, dates d'envol, voltinisme, polygamie, spectre d'hôtes, etc.). Elles se développent sur les mêmes arbres et sont souvent associées lors des dégâts, soit simultanément comme en forêt d'Orléans en 1974-1977 (Vallet, 1982), soit consécutivement, I acuminatus précédant alors de peu I sexdentatus, comme dans le Sud-Est à partir de 1986 (Lieutier et al, 1988a). I acuminatus, de taille inférieure, se localise toutefois dans les zones de l'arbre à écorce fine (branches et partie supérieure du tronc), alors que I sexdentatus préfère les zones à écorce épaisse (partie inférieure du tronc).

\section{MATÉRIELS ET MÉTHODES}

\section{Isolement des champignons}

Les récoltes concernant I acuminatus ont eu lieu dans diverses parcelles forestières du Nord du département du Var, quelque temps après l'attaque, en octobre 1987 , en mai 1988 et en septembre 1988, sur plusieurs arbres tués par cet insecte et ne supportant pas 
d'attaques complémentaires d'/ sexdentatus. Pour une comparaison avec d'autres populations d'/ acuminatus, des récoltes ont aussi eu lieu en forêt d'Orléans en juin 1988 et au Mont Ventoux en juin 1989, sur des rondins, toujours en l'absence d'attaques d'l sexdentatus. En outre, pour une comparaison avec les populations locales d'/ sexdentatus, des récoltes des 2 espèces ont été effectuées dans le Nord du département du Var en octobre 1987, sur des grumes attaquées simultanément par I acuminatus et I sexdentatus; dans le même temps, des récoltes d'/ sexdentatus ont eu lieu dans la même région, sur des grumes ne portant que cette dernière espèce.

Dans chaque cas, les insectes et un fragment de leur galerie ont été prélevés stérilement après écorçage local des arbres attaquées et placés individuellement en tubes stériles à $4{ }^{\circ} \mathrm{C}$ jusqu'à leur utilisation. L'unité de prélèvement était constituée par la galerie et les insectes s'y trouvant. Cette façon de procéder se justifie par le fait que les insectes et leur galerie sont en contact étroit au moment de leur récolte. En plus des prélèvements précédents, des isolements ont eu lieu sur des insectes en essaimage au printemps 1988. Ceux-ci ont été obenus par mise en éclosoir au laboratoire à $20-25^{\circ} \mathrm{C}$, de rondins infestés par $/ \mathrm{acu}$ minatus et prélevés préalablement sur 2 pins de parcelles différentes dans le Nord du département du Var. Les insectes ont alors été récoltés au fur et à mesure de leur émergence et placés individuellement en tubes stériles.

Dans toutes ces opérations, tant sur le terrain qu'au laboratoire, nous nous sommes efforcés de respecter au maximum les conditions de stérilité, en trempant dans l'alcool, puis en flambant tous les instruments d'écorçage et de récolte entre chaque prélèvement, et en évitant les contacts entre insectes provenant de galeries différentes. Les méthodes d'isolement des champignons sur malt-agar à partir des insectes et des galeries ont été décrites précédemment (Lieutier et al, 1989b). Dans le cas des prélèvements après attaques, la présence d'un champignon a été considérée comme effective dans l'unité de prélèvement quand ce champignon apparaissait soit sur un des insectes présents dans la galerie, soit dans la galerie elle-même. Les comparaisons entre fréquences d'association ou entre fréquences de fructification ont été effectuées 2 à 2 par test " $T$ ".

Les identifications ont été réalisées après obtention des formes parfaites (périthèces et ascospores), après s'être assuré de la pureté des colonies par culture monospore. Dans de nombreux cas cependant, seules les formes imparfaites (conidiophores et conidies) ont pu être observées.

\section{Le bleuissement de l'aubier}

À l'automne 1987, des arbres tués par I acuminatus dans le département du Var et situés dans des parcelles différentes, ont été sectionnés transversalement à différentes hauteurs, afin d'y observer l'extension du bleuissement à l'intérieur de l'aubier. Des rondelles de tronc ont en même temps été prélevées sur les mêmes arbres à 2 ou 3 hauteurs différentes au niveau des zones bleuies. Dans la même région et à la même époque, des grumes attaquées par / sexdentatus seul ont aussi été sectionnées et des rondelles y ont été prélevées. Au laboratoire, chaque rondelle a été cassée en plusieurs morceaux; des fragments d'aubier, immédiatement prélevés sur les sections bleuies ainsi mises à jour, ont été mis en culture.

\section{Croissance et aggressivité des champignons}

Après purification par culture monospore sur milieu gélosé à $3 \%$ de malt, les Ophiostoma associés à l acuminatus et isolés des prélèvements varois précédents ont été soumis à des tests de croissance en laboratoire sur le même milieu. Une pastille calibrée de $5 \mathrm{~mm}$ de diamètre, découpée à l'emporte-pièce dans une culture sporulée âgée de 2 semaines, a été déposée mycélium contre gélose, au centre d'une boîte de Petri placée ensuite à $22^{\circ} \mathrm{C}$ à l'obscurité. 4 à 6 répétitions ont été réalisées pour chaque champignon. Tous les jours, l'extension de chaque colonie a été appréciée par la mesure de 2 diamètres orthogonaux entre eux, dont la moyenne a ensuite été calculée après avoir soustrait de chacun d'eux le diamètre de l'implant.

Fin avril 1990, 5 pins sylvestres de $48-56 \mathrm{~cm}$ de circonférence à $1,30 \mathrm{~m}$ de hauteur ont été choisis pour leurs indices de productivité instantanée comparables (rapport de la surface de section du dernier cerne à la surface de section d'aubier à $1,30 \mathrm{~m}$ compris entre 5,0 et $5,7 \%$ ) (Waring et Pitman, 1980). Chacun d'eux a reçu, au niveau du phloème et du cambium, 8 inoculations réparties en 2 couronnes espacées de $40 \mathrm{~cm}$ l'une de l'autre. Les 4 inoculations de chaque couronne correspondaient aux 4 espèces d'Ophiostomatales isolées précédemment dans le Var à partir d'l acuminatus et de ses galeries et qui semblaient correspondre à une réelle association avec cet insecte (cf résultats). Elles correspondaient aussi aux mêmes souches que celles utilisées pour les tests de croissance au laboratoire. La technique d'inoculation dérivée de celle de Wright (1933) a été décrite précédemment (Lieutier et al, 1989b). Trois semaines plus tard, la zone aux alentours de chaque point d'inoculation a été écorcée. Sur la face externe du phloème ainsi mise à jour, on a mesuré la longueur totale de l'imprégnation résinique locale induite dans ce tissu par l'inoculation et matérialisant la réaction de défense de l'arbre à ce niveau. Le quart supérieur droit de cette réaction a été prélevé stérilement pour être rapporté au laboratoire. Des isolements y ont ensuite été effectués tous les $5 \mathrm{~mm}$ à partir du point d'inoculation dans le sens longitudinal, afin d'apprécier les limites du développement du champignon. Les distances obtenues ont été multipliées par 2 pour pouvoir être directement comparées à la longueur totale de réaction. Immédiatement après leur prélèvement, les $3 / 4$ restant ont été placés sous atmosphère 
d'azote et dans la glace carbonique, puis stockés à $-20^{\circ} \mathrm{C}$ au laboratoire jusqu'au dosage de la quantité totale de résine induite. Simultanément à ces récoltes, un échantillon de phloème non réactionnel a été prélevé au niveau de chaque couronne et stocké de la même manière. L'extraction de la résine a été effectuée sur un quart de la réaction en utilisant une technique inspirée de celle de Christiansen (1985) et décrite précédemment (Lieutier et al, 1989b), les 2 derniers quarts étant conservés pour des expérimentations ultérieures. La quantité totale de résine induite contenue dans le quart de réaction a été calculée après avoir soustrait de la quantité totale extraite de ce quart, la quantité de résine contenue dans un échantillon de phloème non réactionnel de même poids et situé à proximité.

Les comparaisons entre champignons pour un paramètre donné (longueur de la réaction induite, extension des champignons ou quantité de résine induite) ont été effectuées par analyse de variance et test de Duncan.

\section{RÉSULTATS}

\section{Nature et fréquence des champignons isolés des insectes et de leurs galeries}

Cinq espèces d'Ophiostomatales ont été isolées $\mathrm{d}^{\prime} /$ acuminatus et de ses galeries : Ophiostoma brunneo-ciliatum Math $-K$, Ophiostoma ips (Rumb) Nannf, Ophiostoma minus (Hedgc) $\mathrm{H}$ et P Syd, un Ophiostoma actuellement à l'étude (dénommé Ophiostoma sp) et Ceratocystiopsis minima (Olchow et Reid) Upadhyay. Deux espèces ont été isolées d'/ sexdentatus et de ses galeries : $O$ brunneo-ciliatum et $O$ ips. La grande majorité des périthèces a été obtenue directement dans les galeries des insectes, une très faible quantité de cultures ayant fourni des formes parfaites. Les cultures n'ayant fourni que des anamorphes correspondaient à des Sporothrix ou à des Hyalorhinocladiella, mais la distinction difficle entre ces 2 formes nous a conduit à les regrouper sous la dénomination de "Sporothrix" prise dans un sens large, sans pouvoir identifier les espèces en cause. Néanmoins, la forme particulière des cultures d' $O$ minus a permis d'identifier ce champignon sous sa forme imparfaite. La forme "Sporothrix" présentée par la suite regroupe donc, selon toute vraisemblance, les 4 autres Ophiostomatales dans le cas $d^{\prime} l$ acuminatus, $O$ brunneo-ciliatum et $O$ ips dans le cas $d^{\prime} /$ sexdentatus.

Les répartitions des différentes formes associées à chacun des 2 Scolytides dans les diverses localités et situations sont présentées dans le tableau I. La fréquence d'association des "Sporothrix" (en y incluant les formes sexuées différant d' $O$ minus) avec les $I$ acuminatus ayant réussi à s'installer est très élevée dans le Var en 1987. Elle diminue de façon très significative en 1988. Les valeurs sont encore plus faibles cette année-là chez les essaimants récoltés dans les mêmes populations juste avant l'attaque. Du point de vue de cette fréquence, les populations varoises d'/ acuminatus en 1988 ne diffèrent pas de celles d'Orléans et du Mont Ventoux. De même, en ce qui concerne la fructification et l'observation des périthèces des champignons associés à I acuminatus, les populations du Var de 1987 diffèrent de celles de 1988, ces dernières présentant les mêmes caractéristiques que les populations d'Orléans et du Mont Ventoux.

En 1987, les populations d'/ acuminatus récoltées dans le Var sur les arbres debout et tués par ce Scolytide, présentent la même fréquence d'association avec les "Sporothrix" et la même composition spécifique en Ophiostomatales, que les populations récoltées sur grumes et comportant en plus des attaques d'/ sexdentatus. L'observation de périthèces dans les galeries est cependant beaucoup plus fréquente sur grume que sur arbre debout. Les diverses populations d'/ sexdentatus récoltées en 1987 dans le Var possèdent des caractéristiques comparables (fréquence des champignons, fructification et composition spécifique de ceux-ci), qu'elles proviennent de grumes indemnes d'attaques $d^{\prime} / \mathrm{acu}$ minatus ou de grumes supportant en plus des attaques d'l Sexdentatus. Comme pour les populations d'/ acuminatus sur grume, elles ont un taux d'association élevé avec les "Sporothrix" et leurs galeries permettent l'observation très fréquente de périthèces. Toutefois, la composition spécifique des Ophiostomatales associées à / sexdentatus diffère toujours beaucoup de la composition spécifique de celles associées à 1 acuminatus, que les 2 espèces d'insectes se trouvent sur le même support ou sur des supports séparés. Dans tous les cas, $O$ minus est très rare, sinon absent.

\section{Caractéristiques du bleuissement de l'aubier}

Sur le tronc des arbres attaqués et tués récemment par I acuminatus dans le Var, il est possible de distinguer 3 zones. Une zone supérieure comprend les branches et la partie du tronc à écorce fine et lisse qui correspond à la zone où se por- 
Tableau I. Fréquence d'association et fructification des Ophiostomatales associées à / acuminatus et / sexdentatus. Sauf mention contraire, il s'agit dans tous les cas d'insectes ayant réussi leurs attaques. (1) Formes sexuées incluses; (2) par rapport au nombre total d'unités de prélèvement; (3) par rapport au nombre d'unités de prélèvement ayant fourni des "Sporothrix"; (4) par rapport au nombre d'unités de prélèvement contenant des fructifications sexuées de "Sporothrix»; $(+)$ un seul cas de fructification ayant été observé, seule est indiquée la nature de l'espèce; $\mathrm{la}=$ I acuminatus; Is : I sexdentatus; $\mathrm{Ob}=\mathrm{O}$ brunneo-ciliatum; $\mathrm{O} \mathrm{i}=\mathrm{O}$ ips; $\mathrm{C} \mathrm{m}=\mathrm{C}$ minima. Les nombres entre parenthèses représentent les intervalles de confiance au seuil de $95 \%$. Deux nombres affectés de la même lettre ne diffèrent pas significativement au seuil de $5 \%$, les comparaisons n'étant effectuées que dans une même ligne (comparaisons 2 à 2 par test «T»). Pour les fructifications sexuées des différentes espèces, chaque lettre est affectée à l'ensemble de la colonne de 4 nombres située en-dessous, les colonnes étant comparées globalement 2 à 2 . Pour chacune de ces colonnes, les différences entre les 4 nombres qui la composent sont globalement hautement significatives (Analyse de variance).

\begin{tabular}{|c|c|c|c|c|c|c|c|c|}
\hline \multicolumn{4}{|c|}{ Var (Octobre 1987) } & \multicolumn{3}{|c|}{$\operatorname{Var}(1988)$} & Orléans & Ventoux \\
\hline I a & $\begin{array}{c}\text { la } \\
\text { (grumes }\end{array}$ & $\begin{array}{l}\text { Is } \\
\text { (grumes }\end{array}$ & $\begin{array}{l}\text { Is } \\
\text { (grumes }\end{array}$ & $\begin{array}{c}\text { avril } \\
\text { I a } \\
\text { essai- }\end{array}$ & $\begin{array}{l}\text { mai } \\
\text { la }\end{array}$ & $\begin{array}{l}\text { septembre } \\
\text { I a }\end{array}$ & $\begin{array}{c}\text { juin } \\
\text { l a } \\
\text { (rondins) }\end{array}$ & $\begin{array}{c}\text { juin } \\
\text { la } \\
\text { (rondins) }\end{array}$ \\
\hline $\begin{array}{c}\text { (arbres } \\
\text { tués) }\end{array}$ & $\begin{array}{l}\text { avec } \\
\text { (s) }\end{array}$ & $\begin{array}{l}\text { avec } \\
\text { la) }\end{array}$ & $\begin{array}{l}\text { sans } \\
\text { la) }\end{array}$ & mants & $\begin{array}{c}\text { (arbres } \\
\text { tués) }\end{array}$ & $\begin{array}{c}\text { (arbres } \\
\text { tués) }\end{array}$ & & \\
\hline
\end{tabular}

\begin{tabular}{|c|c|c|c|c|c|c|c|c|c|}
\hline $\begin{array}{l}\text { Nombre d'arbres } \\
\text { ou de grumes }\end{array}$ & 9 & 5 & 5 & 7 & 2 & 3 & 3 & 1 & 1 \\
\hline $\begin{array}{l}\text { Nombre d'unités } \\
\text { de prélèvement }\end{array}$ & 50 & 15 & 15 & 61 & 281 & 100 & 188 & 22 & 30 \\
\hline $\begin{array}{l}\text { Unités avec } \\
\text { "Sporothrix» (\%) } \\
\begin{array}{ll}\text { (1) } & \text { (2) }\end{array}\end{array}$ & $\begin{array}{c}96 a \\
(81-98)\end{array}$ & $\begin{array}{r}93^{\text {ad }} \\
(68-98)\end{array}$ & $\begin{array}{c}100^{a} \\
(78-100)\end{array}$ & $\begin{array}{c}100^{a} \\
(94-100)\end{array}$ & $\begin{array}{c}49^{b} \\
(43-55)\end{array}$ & $\begin{array}{c}61^{c} \\
(51-71)\end{array}$ & $\begin{array}{c}59 c \\
(52-66)\end{array}$ & $\begin{array}{r}73^{\mathrm{cd}} \\
(50-89)\end{array}$ & $\begin{array}{c}67 \mathrm{bc} \\
(47-83)\end{array}$ \\
\hline
\end{tabular}

Unités avec

\begin{tabular}{|c|c|c|c|c|c|c|c|c|}
\hline O minus (\%) (2) & 0 & 0 & 0 & 0 & 0,4 & 0 & 1,6 & 0 \\
\hline $\begin{array}{l}\text { Fructification } \\
\text { sexuée des } \\
\text { "Sporothrix» (\%) } \\
\text { (3) }\end{array}$ & $\begin{array}{c}38 a \\
(23-51)\end{array}$ & $\begin{array}{c}93^{b} \\
(63-100)\end{array}$ & $\begin{array}{c}100^{b} \\
(78-100)\end{array}$ & $\begin{array}{c}89 b \\
(77-95)\end{array}$ & 0 & 1,6 & 0 & 0 \\
\hline
\end{tabular}

Fructification sexuée des diverses espèces (\%) (4) :

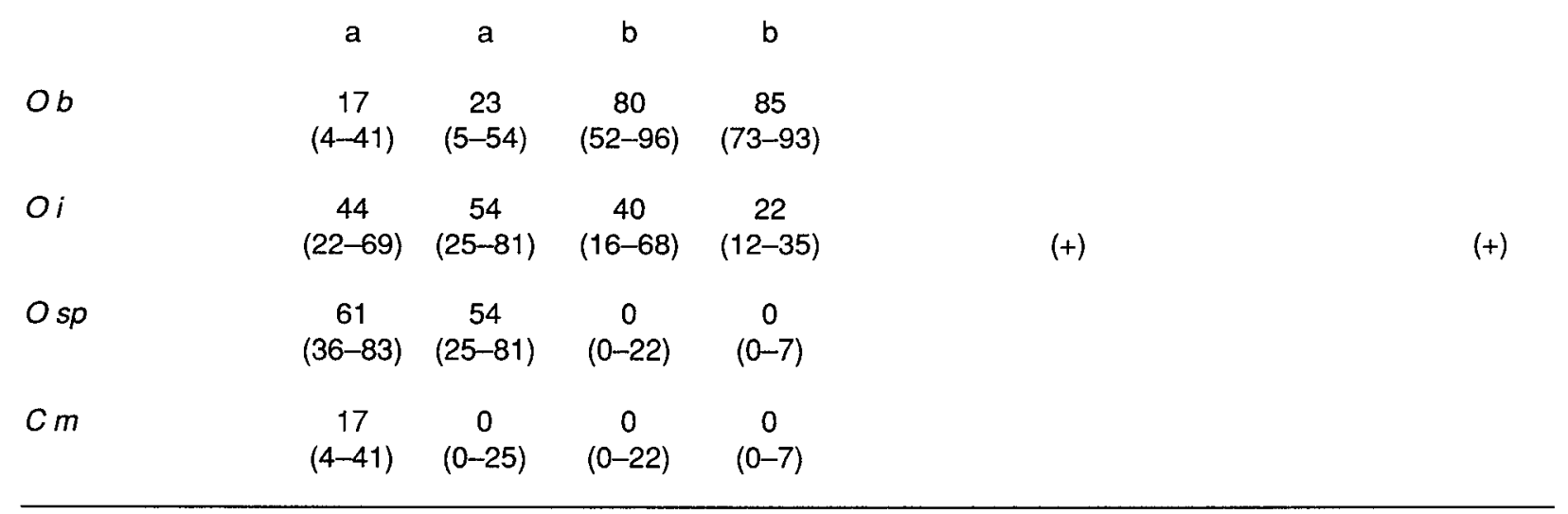

tent naturellement les attaques d'/ acuminatus. C'est dans cette zone que se trouvent les attaques réussies. L'aubier y est entièrement bleu sur toute son épaisseur, tant dans les branches que dans le tronc. Sur les mêmes arbres, une zone médiane correspond à la partie du tronc située dans la zone de transition entre l'écorce lisse et l'écorce fissurée. Elle ne comporte que des attaques avortées d'I acuminatus. L'aubier superficiel montre des réactions résiniques au 
niveau des attaques mais n'est pas bleui. En section transversale, dans l'aubier profond, on observe en général un bleuissement par taches, sans rapport avec la présence des attaques en surface. Sur certains arbres toutefois, la section d'aubier de cette zone apparaît entièrement bleue. Une troisième zone correspond à la partie inférieure du tronc, à écorce fissurée, où aucune attaque n'a lieu. En section transversale, l'aubier profond de cette zone présente parfois, et seulement en profondeur, quelques taches bleues de faibles dimensions.

Sur les grumes attaquées par I sexdentatus dans le Var, la section transversale de l'aubier présente, soit plusieurs taches s'étendant de la partie superficielle jusqu'au bois de cœur, soit une surface entièrement bleuie.

À partir des périthèces obtenus en culture, les isolements effectués sur les divers arbres bleuis ont permis d'identifier $O$ brunneo-ciliatum et $O s p$ dans le cas d'attaques d'I acuminatus; $O$ brunneo-ciliatum seul dans le cas d'attaques d'/ sexdentatus (tableau II). La fréquence d'obtention des "Sporothrix" dans les zones bleuies et le

Tableau II. Fréquence d'obtention et fructification des "Sporothrix" issus de l'aubier bleui des arbres attaqués par I acuminatus ou I sexdentatus dans le Var en octobre 1987. (1) Formes sexuées incluses; (2) par rapport au nombre de rondelles étudiées; (3) par rapport au nombre de rondelles ayant fourni des "Sporothrix"; I a = I acuminatus; I s = I sexdentatus; $\mathrm{O} b=\mathrm{O}$ brunneo-ciliatum. Les nombres entre parenthèses représentent les intervalles de confiance au seuil de $95 \%$.

\begin{tabular}{|c|c|c|}
\hline & la & $\begin{array}{c}\text { Is } \\
\text { (sans I a) }\end{array}$ \\
\hline Nombre d'arbres ou de grumes & 9 & 7 \\
\hline Nombre de rondelles & 21 & 13 \\
\hline $\begin{array}{l}\text { Rondelles avec "Sporothrix" } \\
\begin{array}{lll}(\%) & (1) & (2)\end{array}\end{array}$ & $\begin{array}{c}90 \\
(70-99)\end{array}$ & $\begin{array}{c}100 \\
(75-100)\end{array}$ \\
\hline $\begin{array}{l}\text { Fructification sexuée } \\
\text { des «Sporothrix» (\%) (3) }\end{array}$ & $(3-40)$ & $(2-45)$ \\
\hline \multicolumn{3}{|c|}{ Répartition des formes sexuées (nombre de cas) } \\
\hline $\begin{array}{l}O b \\
O s p\end{array}$ & $\begin{array}{l}2 \\
1\end{array}$ & $\begin{array}{l}2 \\
0\end{array}$ \\
\hline
\end{tabular}

pourcentage de fructification obtenue en culture sont semblables entre les arbres tués par I acuminatus et les grumes supportant les attaques d'I sexdentatus.

\section{Croissance des champignons au laboratoire (fig 1)}

Les souches d'O ips, $O s p$, et $C$ minima ont des vitesses de croissance tout à fait semblables sur malt agar à $22{ }^{\circ} \mathrm{C}$; elles atteignent le bord de la boîte de Petri en 13-14 j. La croissance de la souche d' $O$ brunneo-ciliatum est en revanche un peu plus lente et la boîte de Petri n'est complètement colonisée qu'en 17-18 j.

\section{Agressivité des champignons pour les arbres inoculés (fig 2)}

$O$ ips et $O$ brunneo-ciliatum induisent des réactions significativement plus longues que $O s p$, C minima apparaissant intermédiaire entre ces 2 groupes. Parallèlement, les quantités de résines induites par $O$ ips sont significativement plus importantes que celles induites par $O s p$ et $C$ minima, $O$ brunneo-ciliatum apparaissant dans ce cas intermédiaire. La croissance de ces 4 espèces à l'intérieur des réactions est semblable.

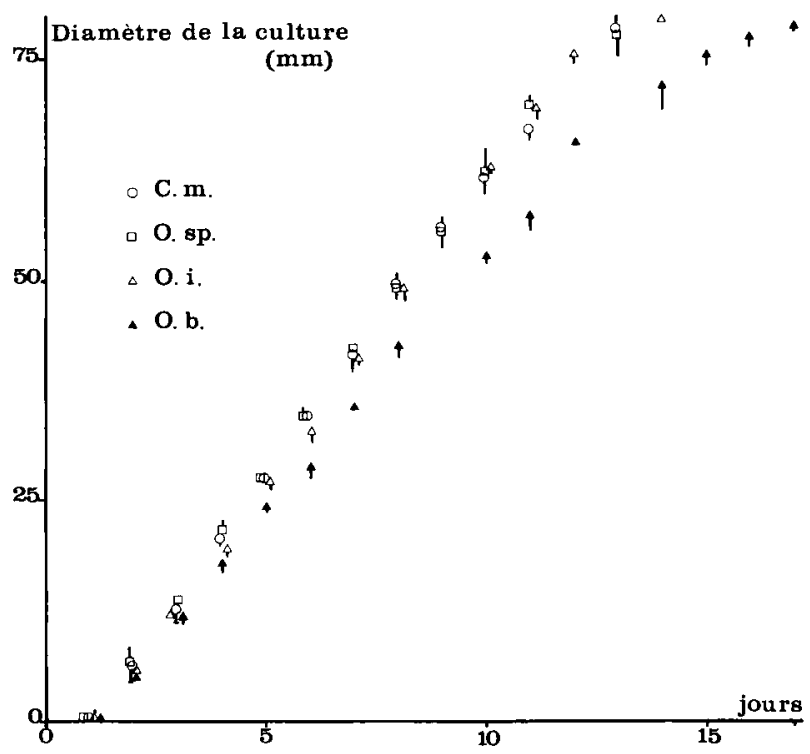

Fig 1. Croissance, à $22^{\circ} \mathrm{C}$ sur malt-agar, des champignons isolés des galeries $\mathrm{d}^{\prime} / \mathrm{acuminatus}$. $\mathrm{Ob}=\mathrm{O}$ brunneo-ciliatum; $\mathrm{Oi}=O \mathrm{ips} ; \mathrm{Cm}=C$ minima . La longueur des barres verticales représente la différence entre la moyenne et la valeur la plus extrême des mesures. 


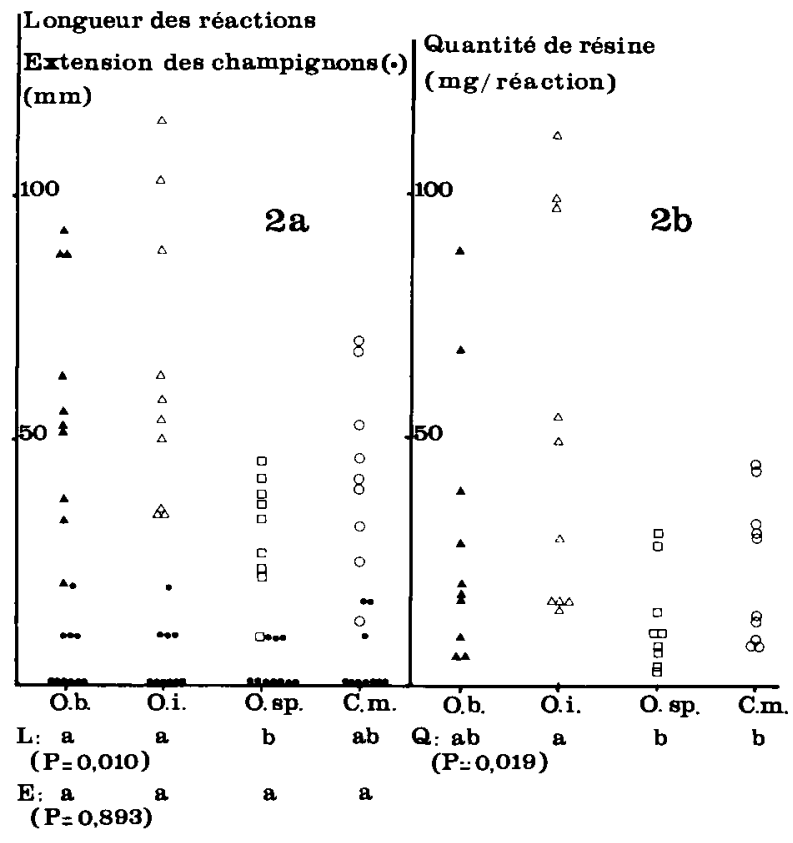

Fig 2. Agressivité des champignons isolés des galeries d'/ acuminatus après inoculation à des pins sylvestres. $2 \mathrm{a}$ : longueur des réactions de défense induites et extension longitudinale des champignons dans ces réactions. $2 \mathrm{~b}:$ quantité de résine dans les réactions de défense induites. $\mathrm{Ob}=0$ brunneo-ciliatum; $\mathrm{Oi}=O$ ips; $\mathrm{Cm}=C$ minima. $\mathrm{L}, \mathrm{Q}, \mathrm{E}=$ résultats des tests statistiques de comparaison entre champignons (analyse de variance et test de Duncan) respectivement pour la longueur de la réaction induite, la quantité de résine induite, l'extension des champignons à l'intérieur de la réaction: deux séries de mesures affectées de la même lettre ne diffèrent pas significativement au seuil de $5 \%$.

\section{INTERPRÉTATION ET DISCUSSION}

\section{Composition spécifique de la flore fongique}

La très faible fréquence et l'irrégularité des isolements d'Ophiostoma minus autorisent à penser que la présence de ce champignon est tout à fait fortuite dans les galeries $\mathrm{d}^{\prime} / \mathrm{l}$ acuminatus. Nous avions été conduits à une conclusion analogue lors d'une précédente étude sur $I$ sexdentatus et $T$ piniperda, bien que $O$ minus puisse être transporté par ce dernier (Lieutier et al, 1989b; Piou et Lieutier, 1989). Mathiesen-Käärik (1960) considère également $O$ minus comme un associé occasionnel de nombreuses espèces d'insectes.

Les investigations menées en Suède et en Allemagne ont mis en évidence, dans ces pays, une association constante et spécifique entre I acuminatus d'une part, Trichosporium tingens var macrosporum et Ceratocystis clavata d'autre part, à laquelle peuvent s'ajouter Ophiostoma pini et un autre Ophiostoma indéterminé (Ren- nerfelt, 1950; Mathiesen, 1950; FranckeGrosmann, 1952, 1963b; Mathiesen-Käärik, 1953; Henningsson et Lundström, 1974). Aucune de ces espèces n'a été observée dans nos relevés. Bien que Francke-Grosmann (1952, 1966) considère $T$ tinges comme un véritable symbionte utilisé dans l'alimentation des larves âgées et des jeunes adultes d'/ acuminatus, l'association entre ces 2 organismes ne paraît donc pas obligatoire. En revanche, nous avons trouvé dans les galeries d'l acuminatus, $O$ brunneociliatum, considéré comme spécifique d'l sexdentatus (Lieutier et al, 1989b), ainsi que $C$ minima. La présence de ce dernier pourrait peut être s'expliquer par la date un peu tardive des prélèvements (octobre) par rapport à la date d'attaque des insectes (août), puisque Olchowecki et Reid (1974) le considèrent plutôt comme secondaire. $O$ ips est aussi représenté dans nos relevés, mais ce champignon a une très vaste répartition géographique et Francke-Grosmann (1966) signale qu'il est associé à de très nombreuses espèces de scolytides dans toute la zone paléarctique. Néanmoins, il n'avait pas encore été signalé chez I acuminatus. La flore fongique associée à l acuminatus semble donc présenter des différences très importantes selon les localités, au moins quand on compare 2 situations extrêmes de l'aire de répartition de ce scolytide. La flore associée à I sexdentatus paraît plus stable, puisque les espèces trouvées dans cette étude sont les mêmes que celles observées dans la région d'Orléans (Lieutier et al, $1989 b)$ et en Suède (Mathiesen, 1950).

Il est intéressant de remarquer que $O$ ips et O brunneo-ciliatum, couramment associés à I sexdentatus, font partie ici du cortège de champignons isolés d'/ acuminatus. Étant donné la présence possible des 2 scolytides sur les mêmes arbres, un passage de la flore d'une espèce à l'autre a pû intervenir. Toutefois, un tel phénomène n'a pas été signalé en Suède où les 2 espèces coexistent pourtant (Rennerfelt, 1950; Mathiesen, 1950; Mathiesen-Käärik, 1953). En outre, dans les prélèvements effectués sur les grumes attaquées simultanément par les 2 insectes, les fréquences des divers champignons associés diffèrent significativement d'une espèce de scolytide à l'autre; les rapports entre les fréquences d'O ips et d'O brunneo-ciliatum y sont même inverses. Au contraire, pour une même espèce d'insecte, il n'y a aucune différence à ce sujet, selon qu'elle se trouve seule ou en présence de l'autre. Chacune des 2 espèces de scolytides semble donc avoir une composi- 
tion fongique particulière, même quand il s'agit d'espèces communes de champignons. Pour chacune des 2 espèces $O$ ips et $O$ brunneociliatum, on peut alors s'interroger sur l'identité des souches associées aux 2 scolytides. Mathiesen-Käärik (1960) a, de même, rapporté l'existence d'associations fongiques différentes pour $I$ acuminatus et Tomicus minor alors que ces 2 espèces évoluent sur les mêmes arbres et dans les même zones.

\section{Évolution de la fréquence d'association insecte-champignon}

Très élevée en 1987, la fréquence d'association des populations épidémiques $\mathrm{d}^{\prime} l$ acuminatus avec les "Sporothrix» diminue de façon très significative dans le Var en 1988, jusqu'à des valeurs comparables avec ce que l'on observe dans les populations endémiques du Mont Ventoux et de l'Orléanais. Parallèlement, alors que le taux de fructification sexuée de ces "Sporothrix" atteint $38 \%$ sur les arbres tués en 1987, il devient presque nul en 1988, comme au Mont Ventoux et en Orléanais. Or, les taches de dépérissement ont diminué dans les peuplements de pins sylvestres du Var à partir de 1989, d'abord légèrement, puis très nettement en 1990, malgré la sécheresse. II est, dans ces conditions, très tentant d'établir un lien entre l'évolution des caractéristiques de la population de champignons et la diminution des dégâts qui ont suivi cette évolution. Une population fongique dynamique, présentant un taux de présence élevé dans la population d'insectes et un fort taux de fructification sexuée, pourrait ainsi correspondre à de hauts niveaux de population de ravageurs. Les résultats de cette étude sont cependant trop limités pour conclure en ce sens et a fortiori pour proposer des hypothèses explicatives. II est possible aussi que les fréquences d'association aient été surestimées en 1987, si $C$ minima, à tendance secondaire d'après Olchowecki et Reid (1974), a pû être présent sans avoir été apporté par les insectes.

Rumbolt (1941) a signalé que la composition spécifique de la flore fongique associée à Dentroctonus ponderosae, ainsi que la vitesse de croissance en culture des champignons la composant, différaient selon que les populations de ce scolytide se trouvaient en phase endémique ou épidémique. Bridges (1985) suggère que l'absence de bleuissement du bois des arbres attaqués par Dendroctonus frontalis pourrait être in- dicateur d'une tendance des populations à pulluler.

\section{Rôle des champignons dans la réussite des attaques}

Le fait que les "Sporothrix" soient, de façon significative, moins fréquents sur les $l$ acuminatus en essaimage que sur ceux qui sont installés sur les arbres, suggère que la présence de ces champignons favorise le succès des attaques. Or, la réponse des arbres à des inoculations artificielles de champignons, appréciée à la fois par la longueur de la réaction et la quantité de résine induite, permet de juger de l'agressivité de ces derniers pour l'hôte inoculé (Lieutier et al, 1990). Les résultats obtenus dans cette étude conduisent, de cette manière, à séparer les champignons étudiés en 2 groupes d'agressivité différente : $O$ ips et $O$ brunneo-ciliatum d'une part, $O$ sp et $C$ minima d'autre part (fig 2). Les 2 premiers nommés, plus agressifs, semblent donc les meilleurs candidats pour jouer un rôle favorisant le succès des attaques d'/ acuminatus, bien que $O$ brunneo-ciliatum pousse moins vite sur milieu gélosé (fig 1).

En effet, on sait qu'il existe pour chaque arbre un seuil de densité d'attaques au-delà duquel il succombe et que la valeur de ce seuil dépend en grande partie de l'état physiologique du sujet (Berryman, 1982; Raffa et Berryman, 1983; Christiansen et Horntvedt, 1983; Waring et Pitman, 1983; Mulock et Christiansen, 1986; Christiansen et al, 1987; Lieutier et Ferrell, 1988). En favorisant la synthèse de grandes quantités de résine dont la fabrication épuise les ressources énergétiques de l'hôte, $O$ ips et $O$ brunneociliatum pourraient donc abaisser la valeur du seuil critique de densité d'attaques par I acuminatus, comme dans le cas d'/ sexdentatus (Lieutier, 1991). $O s p$ et $C$ minima poussent aussi bien que $O$ ips en l'absence de résistance de l'hôte, mais ils n'induisent qu'une faible réaction quand ils sont inoculés à l'arbre. Ils ne peuvent donc sans doute que très peu contribuer au succès de l'installation d'l acuminatus.

\section{Le bleuissement de l'aubier et son évolution}

Les résultats du tableau II montrent que les "Sporothrix" sont associés au bleuissement de l'aubier. Chez I acuminatus, seuls $O$ brunneo- 
ciliatum et $O s p$ ont été identifiés parmi les formes sexuées obtenues au laboratoire à partir des zones bleuies, mais le faible nombre de cas de fructification n'autorise pas à conclure qu'ils sont seuls impliqués dans le bleuissement. Les observations réalisées sur la répartition des zones bleuies permettent de conclure que la présence du bleu dans l'aubier est directement liée à la réussite des attaques d'l acuminatus. A partir de la région supérieure de l'arbre (branches et zone du tronc à écorce fine) envahie la première en totalité, les champignons présents dans l'aubier et le bleuissement qui les accompagne s'étendraient à l'arbre entier en descendant vers sa base par la partie la plus interne de l'aubier. Ils envahiraient ensuite la totalité de ce tissu en rayonnant vers sa périphérie.

Le déroulement de ce processus expliquerait la nature des symptômes rapportés précédemment (Lieutier et al, 1988a). Le jaunissement du feuillage, quelques semaines après le succès des attaques des populations épidémiques d'l acuminatus, serait la conséquence d'un arrêt de l'alimentation en eau de la cime dû à l'obstruction complète de l'aubier par les champignons dans la moitié supérieure du tronc, la moitié inférieure restant, pendant ce temps, apparemment saine. Le blocage mécanique de la circulation de l'eau dans l'aubier de divers conifères lors d'attaques par des Ophiostoma, associés ou non à des Scolytides, a déjà été évoqué par d'autres auteurs (Mathre, 1964; Basham, 1970; Horntvedt et al, 1983; entre autres). L'intervention de toxines sécrétées par les champignons et perturbant les échanges hydriques peut cependant aussi être avancée, comme dans le cas de $O$ minus sur Pinus taeda (Hodges et al, 1985a, b). Dans le cas des attaques $\mathrm{d}^{\prime} /$ acuminatus sur pin sylvestre, le mode de progression des champignons et du bleu vers le bas de l'arbre permettrait la persistence de l'alimentation en eau de cette zone pendant un certain temps, au moins jusqu'à la saison suivante de vol des Scolytides (mai ou août selon l'époque de la première attaque). À ce moment, l'arbre présenterait pour I sexdentatus, dont les attaques se localisent dans la moitié inférieure du tronc, toutes les caractéristiques d'un arbre sain, mais ses capacités de défense dans cette zone seraient très diminuées à cause de l'absence de ressources énergétiques produites par la photosynthèse. Les attaques de ce scolytide pourraient alors réussir, bien que ces populations soient endémiques, comme cela a été observé sur certains arbres (Lieutier et al, 1988a). Les champignons appor- tés par I sexdentatus envahiraient alors l'aubier à partir de sa périphérie, contribuant à son bleuissement rapide.

\section{REMERCIEMENTS}

Le travail présenté a pu être réalisé grâce à un financement du Conseil régional de la région PACA. Les auteurs expriment toute leur gratitude à l'Office national des forêts et particulièrement à $T$ Faure de Comps-sur-Artuby (Var) pour les facilités de terrain qui leur ont été offertes. lis remercient aussi le Dr WJ Mattson d'avoir bien voulu relire et corriger le résumé anglais.

\section{RÉFÉRENCES}

Basham HG (1970) Wilt of loblolly pine inoculated with blue-stain fungi of the genus Ceratocystis. Phytopathology, 60, 750-754

Berryman AA (1982) Biological control, thresholds and pest outbreaks. Environ Entomol 11, 544-549

Bridges JR (1985) Relationship of symbiotic fungi to southern pine beetle population trends. In: Proc $/ n$ tegrated Pest Manage Res Symp, Ashville (USA), Gen Tech Rep SO-56, SFES, Nex-Orleans, 127135

Christiansen E (1985) Ceratocystis polonica inoculated in Norway spruce: blue-staining in relation to inoculum density, resinosis and tree growth. Eur $J$ For Pathol 15, 160-167

Christiansen E, Horntvedt R (1983) Combined Ips/ Ceratocystis attack on Norway spruce, and defence mechanisms of the trees. $Z$ Angew Entomol 96, 110-118

Christiansen E, Waring RH, Berryman AA (1987) Resistance of conifers to bark beetle attack: searching for general relationship. For Ecol Manage 22, 89106

Francke-Grosmann H (1952) Über die Ambrosiazucht der beiden Kiefernborkenkäfer Myelophilus minor $\mathrm{Htg}$ und Ips acuminatus Gyll. Medd Statens Skogsförsöksant 41, 1-52

Francke-Grosman H (1963a) Some new aspects in forest entomology. A Rev Entomol 8, 415-438

Francke-Grosmann H (1963b) Die Übertragung der Pilzflora bei dem Borkenkäfer Ips acuminatus Gyll. Ein Beitrag zur kenntniss der Ipiden-Symbiosen. $Z$ Angew Entomol 52, 355-361

Francke-Grosmann H (1966) Über Symbiosen von xylomycetophagen und phloeophagen Scolytoïdea mit holzbewohnenden Pilzen. Int Symp "Holz und Organismen", Berlin-Dahlen, Berlin, Duncker \& Humblot 1, 503-522

Francke-Grosmann H (1967) Ectosymbiosis in woodinhabiting insects. In: Symbiosis, vol 2, SM Henry (ed), Acad Press, New York, 141-205 
Graham K (1967) Fungal-insect mutualism in trees and timber. Annu Rev Entomol 12, 105-126

Henningsson B, Lundström H (1974) The growth of insect-born bluestain, and effects caused by immersion of the wood; some laboratory tests. Rapporter, Institutionen for Virkeslära, R 92, $20 \mathrm{p}$

Hodges JD, Nebeker TE, De Angelis JD, Karr BL, Blanche CA (1985a) Host resistance and mortality: A hypothesis based on the southern pine beetle microorganism - host interactions. Bull Entomol Soc Am 21, 31-35

Hodges JD, Nebeker TE, De Angelis JD, Blanche CA (1985b) Host/Beetle interactions: Influence of associated microorganisms, tree disturbance, and host vigor. Proc Integr Pest Manage Res Symp, Asheville (USA), Gen Tech Rep SO-56, SFES, New Orleans, 161-168

Horntvedt R, Christiansen E, Solheim H, Wang SG (1983) Artificial inoculation with lps typographus associated blue-stain fungi can kill healthy Norway spruce trees. Medd Nor Inst Skogforsk 38, 1-20

Langström B, Hellqvist C, Ericsson A, Gref R (1990) Defense reaction of Scots pine against attacks by Tomicus piniperda. XIXth IUFRO World Congress, Montréal (Canada), 5-11 august

Lieutier F (1991) Les réactions de défense des conifères et stratégies d'attaque de quelques Scolytides européens. Actes $3^{e}$ Conf Int Entomologistes d'Ex pression Française, Gembloux (Belgique), 9-14 juillet (sous presse)

Lieutier F. Ferrell GT (1988) Relationships between indexes of tree vigour and the induced defense reaction of Scots pine to a fungus associated with Ips sexdentatus (Coleoptera: Scolytidae). In: Integrated Control of Scolytid Bark Beetles (Payne TL, Saarenmaa $\mathrm{H}$, eds), Virg Polytech Inst St Univ, 163178

Lieutier F, Faure T, Garcia J (1988a) Les attaques de Scolytes et le dépérissement du pin sylvestre dans la région Provence-Côte d'Azur. Rev For Fr 40, 224-232

Lieutier F, Yart A, Garcia J, Poupinel B, Levieux J (1988b) Do fungi influence the establishment of bark beetles in Scots pine ? In: Mechanisms of woody plant defenses against insects (Mattson WJ Levieux J, Bernard-Dagan C, eds), Springer, New York, 317-330

Lieutier F, Cheniclet C, Garcia J (1989a) Comparison of the defense reaction of Pinus pinaster and Pinus sylvestris to attacks by two bark beetles (Coleoptera: Scolytidae) and their associated fungi. Environ Entomol 18, 228-234

Lieutier F, Yart A, Garcia J, Ham MC, Morelet M, Levieux $J(1989 b)$ Champignons phytopathogènes associés à Ips sexdentatus Boern et Tomicus piniperda $\mathrm{L}$ (Coleoptera : Scolytidae) et étude préliminaire de leur agressivité pour le pin sylvestre. Ann $\mathrm{Sci}$ For 46, 201-216
Lieutier F, Yart A, Garcia J, Ham MC (1990) Cinétique de croissance des champignons associés à lps sexdentatus Boern et à Tomicus piniperda $\mathrm{L}$ (Coleoptera: Scolytidae) et des réactions de défense des pins sylvestres (Pinus sylvestris $L$ ) inoculés. Agronomie 10, 243-256

Mathiesen A (1950) Über einige mit Borkenkäfer assozilerte Bläuepilze in Schweden. Oïkos 2, 275-308

Mathiesen-Käärik A (1953) Eine Übersicht über die gewöhnlichsten mit Borkenkäfern assoziierten Bläuepilze in Schweden und einige für Schweden neue Blänepilze. Medd Stat Skogforsk 43, 1-74

Mathiesen -Käärik A (1960) Studies on the ecology, taxonomy and physiology of Swedish insectassociated blue-stain fungi, especially the genus Ceratocystis. Oikos 11, 1-25

Mathre (de) (1964) Effect of Ceratocystis ips and $\mathrm{Ce}$ ratocystis minor on the free sugar pool in ponderosa pine sapwood. Contrib Boyce Thompson Inst 22 , 509-511

Mulock P, Christiansen E (1986) The threshold of successful attack by Ips typographus on Picea abies: a field experiment. For Ecol Manage 14, 125-132

Olchowecki A, Reid J (1974) Taxonomy of the genus Ceratocystis in Manitoba. Can J Bot 52, 1675-1711

Piou D, Lieutier F (1989) Observations symptomatologiques et rôle possible d'Ophiostoma minus Hedgc (Ascomycete : Ophiostomatales) et de Tomicus piniperda $L$ (Coleoptera : Scolytidae) dans le dépérissement du pin sylvestre en forêt d'Orléans. Ann Sci For 46, 39-53

Raffa KF, Berryman AA (1983) The role of host resistance in the colonization behavior and ecology of bark beetles (Coleptera: Scolytidae). Ecol Monog $53,27-49$

Rennerfelt E (1950) Über den Zusammenhang zwischen dem Verblauen des Holzes und den Insekten. Oikos 2, 120-137

Rumbold CT (1941) A blue stain fungus, Ceratostomella montium $\mathrm{n} \mathrm{sp}$ and some yeasts associated with two species of Dendroctonus. J Agric Res 62 , 589-601

Solheim H (1986) Species of Ophiostomataceae isolated from Picea abies infected by the bark beetle lps typographus. Nord J Bot 6, 199-207

Solheim H, Langström B (1991) Blue-stain fungi associated with Tomicus piniperda in Sweden and preliminary observations on their pathogenecity. Ann Sci For 48, 149-156

Vallet $E$ (1982) Données bioécologiques récentes sur trois insectes ravageurs responsables du dépérissement du pin sylvestre en Région Centre : Tomicus piniperda, lps acuminatus et lps sexdentatus (Coleoptera : Scolytidae). Bull Inst Ecol Appl Orléans 1/2, 3-41 
Waring RH, Pitman GB (1980) A simple model of host resistance to bark beetles. Oreg St Univ, For Res Lab, Res Note 65, 2 p

Waring RH, Pitman GB (1983) Physiological stress in lodgepole pine as a precursor for mountain pine beetle attack. $Z$ Angew Entomol 96, 265-270
Whitney HS (1982) Relationships between bark beetles and symbiotic organisms. In: Bark beetles in North American Conifers (Mitton JB, Sturgeon KD, eds) Univ Tex Press, Austin, 183-211

Wright $E$ (1983) A cork-borer method for inoculating trees. Phytopathology 23, 487-488 